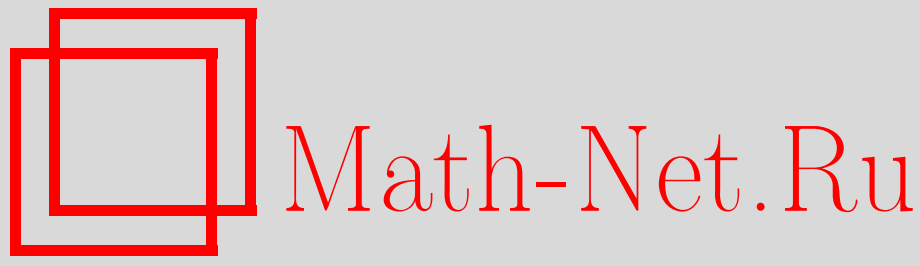

Общероссийский математический портал

Н. А. Сидорова, Предельное поведение поверхностных мер на пространствах траекторий, Матем. заметки, 2004, том 76, выпуск 2, 307-311

DOI: https://doi.org/10.4213/mzm571

Использование Общероссийского математического портала Math-Net.Ru подразумевает, что вы прочитали и согласны с пользовательским соглашением http://www.mathnet.ru/rus/agreement 
Параметры загрузки:

IP: 54.80 .73 .141

26 апреля 2023 г., 14:44:18

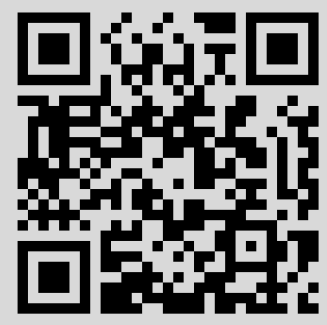




\section{ПРЕДЕЛЬНОЕ ПОВЕДЕНИЕ ПОВЕРХНОСТНЫХ МЕР НА ПРОСТРАНСТВАХ ТРАЕКТОРИЙ}

\section{Н. А. Сидорова}

Введение. В работе рассматриваются поверхностные меры на пространстве траекторий $C([0, T], M)$ в компактном римановом многообразии $M$, вложенном в объемлющее пространство (евклидово пространство $\mathbb{R}^{n}$ или другое многообразие). Эти меры (называемые также $n о в е р x-$ ностными мерами Смолянова) были впервые введены в [1] и затем исследовалось в [2] для случая $T=1$. В данной работе описываются эти меры для произвольного $T$, а также исследуется их сходимость при $T \rightarrow \infty$ в случае, когда $M$ одномерно.

1. Поверхностные меры для произвольных интервалов времени. Пусть $M$ - компактное риманово многообразие, вложенное в $\mathbb{R}^{n}$ и $a_{0} \in M$ - фиксированная точка. Пусть $\mathbb{M}_{\varepsilon}=$ $\left\{a \in \mathbb{R}^{n}: \operatorname{dist}(a, M) \leqslant \varepsilon\right\}$ - трубчатая $\varepsilon$-окрестность многообразия $M$, где dist обозначает евклидову метрику в $\mathbb{R}^{n}$.

Пусть $A$ - одно из множеств $\mathbb{R}^{n}, M$ или $\mathbb{M}_{\varepsilon}$. Обозначим $C_{a_{0}}([0, T], A)$ пространство непрерьвных функций из $[0, T]$ в $A$, равных $a_{0}$ в нуле.

Пусть $\left(b_{t}\right)$ - броуновское движение в $\mathbb{R}^{n}$ с началом в точке $a_{0}$. Обозначим символом $\mathbb{W}_{\varepsilon, T}$ вероятностную меру на пространстве $C_{a_{0}}\left([0, T], \mathbb{R}^{n}\right)$ (сконцентрированную на множестве $C_{a_{0}}([0, T]$, $\left.\mathbb{M}_{\varepsilon}\right)$, , являющуюся условным распределением случайного процесса $\left(b_{t}\right)$ при условии, что он не покидает трубчатую $\varepsilon$-окрестность многообразия вплоть до времени $T$, т.е.

$$
\mathbb{W}_{\varepsilon, T}=\frac{\left.\mathbb{W}_{T}\right|_{C_{a_{0}}\left([0, T], \mathbb{M}_{\varepsilon}\right)}}{\mathbb{W}_{T}\left(C_{a_{0}}\left([0, T], \mathbb{M}_{\varepsilon}\right)\right)}
$$

где $\mathbb{W}_{T}$ - стандартная мера Винера на $C_{a_{0}}\left([0, T], \mathbb{R}^{n}\right)$.

ОПРЕДЕЛЕниЕ 1 . Слабый предел $\mathbb{S}_{T}$ семейства мер $\mathbb{W}_{\varepsilon, T}$ при $\varepsilon \rightarrow 0$ назьвается поверхностной мерой на пространстве $C_{a_{0}}([0, T], M)$. 
Обобщая результаты [2] на случай произвольного $T$, мы получаем, что эта мера существует, абсолютно непрерывна относительно меры Винера $\mathbb{W}_{T}^{M}$ на пространстве путей в многообразии до времени $T$, и ее плотность Радона-Никодима задается формулой

$$
\frac{d \mathbb{S}_{T}}{d \mathbb{W}_{T}^{M}}(\omega)=\frac{\exp \left\{-\frac{1}{4} \int_{0}^{T} R\left(\omega_{s}\right) d s+\frac{1}{8} \int_{0}^{T}\|\sigma\|^{2}\left(\omega_{s}\right) d s\right\}}{\mathbb{E}_{\mathbb{W}_{T}^{M}} \exp \left\{-\frac{1}{4} \int_{0}^{T} R\left(\omega_{s}\right) d s+\frac{1}{8} \int_{0}^{T}\|\sigma\|^{2}\left(\omega_{s}\right) d s\right\}},
$$

где $R$ - скалярная кривизна, а $\sigma$ - вектор средней кривизны многообразия, умноженный на его размерность. В случае, когда $M$ - компактное одномерное многообразие (т.е. замкнутая кривая) в $\mathbb{R}^{n}$, эта формула записьвается в виде

$$
\frac{d \mathbb{S}_{T}}{d \mathbb{W}_{T}^{M}}(\omega)=\frac{\exp \left[\int_{0}^{T} v\left(\omega_{s}\right) d s\right]}{\mathbb{E}_{\mathbb{W}_{T}^{M}} \exp \left[\int_{0}^{T} v\left(\omega_{s}\right) d s\right]}, \quad \text { где } v(a)=\frac{1}{8} \kappa^{2}(a)
$$

а $\kappa$ - кривизна кривой.

Далее мы рассматриваем случай $\operatorname{dim} M=1$. Обозначим через $\left(y_{t}^{T}\right), t \leqslant T$, случайный процесс в $M$ с началом в $a_{0}$, соответствуюший мере $\mathbb{S}_{T}$.

Теорема 1. Для каждого $T$ случайный прочесс $\left(y_{t}^{T}\right), t \leqslant T$, удовлетворяет стохастическому дифференциальному уравнению

$$
d y_{t}^{T}=d b_{t}+(\nabla \log u)\left(T-t, y_{t}^{T}\right) d t
$$

с начальным условием $y_{0}^{T}=a_{0}$, әде $и$-решение уравнения

$$
\partial_{t} u=\frac{1}{2} \Delta u+v u
$$

с начальным условием $u(0, x)=1$ для всех $x$.

ДоКАЗАТЕЛЬСтво. Заметим, что вместо мер, процессов и уравнений, соответствующих замкнутой кривой, можно рассматривать их периодические аналоги, соответствующие $\mathbb{R}$. По формуле Фейнмана-Каца

$$
u(t, x)=\mathbb{E}_{W_{T}^{x}} \exp \left[\int_{0}^{t} v\left(\omega_{s}\right) d s\right],
$$

где $W_{T}^{x}$ - мера Винера на $C_{x}([0, T], \mathbb{R})$. Рассмотрим $\left(y_{t}^{T}\right)$ как координатный процесс $\left(\omega_{t}\right)$ навероятностном пространстве $\left(C_{0}([0, T], \mathbb{R}), \mathscr{F}_{t}, \mathbb{S}_{T}\right)$. Так как $\left(\omega_{t}\right)$ - броуновское движение относительно меры Винера $\mathbb{W}_{T}^{0}$, эквивалентной мере $\mathbb{S}_{T}$, то мы получаем по теореме Гирсанова $d \omega_{t}=d b_{t}+$ $d r_{t} d \omega_{t} / r_{t}$, где

$$
\begin{aligned}
r_{t} & =\mathbb{E}\left[d \mathbb{S}_{T} / d \mathbb{W}_{T}^{0} \mid \mathscr{F}_{t}\right]=\frac{\mathbb{E}\left[\exp \left(\int_{0}^{T} v\left(\omega_{s}\right) d s\right) \mid \mathscr{F}_{t}\right]}{u(T, 0)} \\
& =\frac{\exp \left(\int_{0}^{t} v\left(\omega_{s}\right) d s\right) \mathbb{E}\left[\exp \left(\int_{t}^{T} v\left(\omega_{s}\right) d s\right) \mid \mathscr{F}_{t}\right]}{u(T, 0)}=\frac{\exp \left[\int_{0}^{t} v\left(\omega_{s}\right) d s\right] u\left(T-t, \omega_{t}\right)}{u(T, 0)}
\end{aligned}
$$

и, следовательно,

$$
\begin{aligned}
\frac{d r_{t} d \omega_{t}}{r_{t}} & =\frac{\left(\exp \left[\int_{0}^{t} v\left(\omega_{s}\right) d s\right] \nabla u\left(T-t, \omega_{t}\right) d t\right) / u(T, 0)}{\left(\exp \left[\int_{0}^{t} v\left(\omega_{s}\right) d s\right] u\left(T-t, \omega_{t}\right)\right) / u(T, 0)} \\
& =\nabla \log u\left(T-t, \omega_{t}\right) d t
\end{aligned}
$$

что приводит к искомому коэффициенту сноса в уравнении (1). 
2. Сходимость при $T \rightarrow \infty$. Заметим, что для различных $T$ меры $\mathbb{S}_{T}$ определены на разных пространствах $C_{a_{0}}([0, T], M)$ и доопределим их (произвольно) до счетно-аддитивных мер на общем пространстве $C_{a_{0}}([0, \infty], M)$.

Лемма 1. Линейный оператор $\frac{1}{2} \Delta+v$ обладает ровно одним собственным значением $с$, обладающим тем свойством, что соответствующая ему собственная функиия $\varphi$ полохительна. В этом случае $\varphi$ единственна с точностью до умножения на полохительное число.

ДокАЗАТЕЛЬСтво. Мы снова будем рассматривать периодическую интерпретацию в $\mathbb{R}$. B этом случае искомая собственная функция должна быть не только положительной, но еще и периодической.

Обозначим

$$
L=\frac{1}{2} \Delta+v \quad \text { и } \quad C_{L}(\mathbb{R})=\left\{u \in C^{2}(\mathbb{R}): L u=0 \text { и } u>0\right\} .
$$

Согласно [3] (см. также [4]) для каждого $\lambda \in \mathbb{R}$ обозначим

$$
\begin{aligned}
\Gamma_{\lambda}= & \left\{\nu \in \mathbb{R}: \exists u \in C_{L-\lambda}(\mathbb{R}), \text { имеющая вид } u(t)=e^{\nu t} \psi_{\nu}(t),\right. \\
& \text { где } \left.\psi_{\nu}-\text { периодическая функция }\right\}, \\
K_{\lambda}= & \left\{\nu \in \mathbb{R}: \exists u \in C^{2}(\mathbb{R}) \text { такая, что }(L-\lambda) u \leqslant 0 \text { и } u>0,\right. \\
& \text { и имеющая вид } \left.u(t)=e^{\nu t} \psi_{\nu}(t), \text { где } \psi_{\nu}-\text { периодическая функция }\right\} .
\end{aligned}
$$

По теореме A1 из [3] существует действительное число $\lambda^{*}$ такое, что

1) если $\lambda=\lambda^{*}$, то $\Gamma_{\lambda}=K_{\lambda}=\left\{\nu_{0}\right\}$ с некоторым $\nu_{0} \in \mathbb{R}$;

2) если $\lambda<\lambda^{*}$, то $\Gamma_{\lambda}=K_{\lambda}=\varnothing$;

3) если $\lambda>\lambda^{*}$, то $K_{\lambda}$ строго вьпукло и компактно, а $\Gamma_{\lambda}=\partial K_{\lambda}$;

4) $K_{\lambda_{1}} \subsetneq K_{\lambda_{2}}$ для всех $\lambda^{*} \leqslant \lambda_{1}<\lambda_{2}$

5) $K_{\lambda}^{*}=-K_{\lambda}$, где $K^{*}$ соответствует сопряженному оператору $L^{*}$.

Далее, согласно следствию А3 из [3] для каждой функции $u \in C_{L-\lambda}(\mathbb{R})$ существует единственная конечная мера $\mu_{u}$ на множестве $\Gamma_{\lambda}$ такая, что

$$
u(t)=\int_{\Gamma_{\lambda}} e^{\nu t} \psi_{\nu}(t) \mu_{u}(d \nu)
$$

где для каждого $\nu \in \Gamma_{\lambda}$ соответствующая функция $\psi_{\nu}$ является периодической. Поскольку в нашем случае оператор $L$ является самосопряженньм, мы имеем $K_{\lambda^{*}}=-K_{\lambda^{*}}$ и, следовательно, $\nu_{0}=0$ согласно свойству 5$)$.

Пусть $c=\lambda^{*}$ и $u \in C_{L-c}(\mathbb{R}) ;$ по формуле (3) и свойствам 1) и 5$) u(x)=K \psi_{\nu_{0}}(x)$, где $K>0-$ некоторая константа. Следовательно, множество положительных собственных функций оператора $\frac{1}{2} \Delta+v$ с собственным значением $c=\lambda^{*}$ состоит из единственной (с точностью до умножения на положительное число) функции, которая, кроме того, является периодической.

Если $c<\lambda^{*}$, то $\Gamma_{c}=\varnothing$ согласно свойству 2$)$, и $C_{L-c}(\mathbb{R})=\varnothing$ вследствие (3). Если же $c>\lambda^{*}$, то по лемме 3 из [3] $\Gamma_{\lambda}=\left\{\nu \in \mathbb{R}: \lambda_{0}(\nu)=\lambda\right\}$, где $\lambda_{0}$ - некоторая непрерывная функция. Следовательно, $0=\nu_{0} \notin \Gamma_{c}$. Согласно свойствам 3$)$ и 4$)$ мы получаем $K_{c}=\left[a_{c}, b_{c}\right]$, где $a_{c}<0<b_{c}$, и $\Gamma_{c}=\left\{a_{c}, b_{c}\right\}$. Пусть $u \in C_{L-c}(\mathbb{R})$, тогда

$$
u(t)=e^{a_{c} t} \psi_{a_{c}}(t) \mu\left(\left\{a_{c}\right\}\right)+e^{b_{c} t} \psi_{b_{c}}(t) \mu\left(\left\{b_{c}\right\}\right)
$$

вследствие (3), и, следовательно, $u$ не может быть периодической. Таким образом, у оператора $\frac{1}{2} \Delta+v$ не существует положительных периодических собственных функций с собственными значениями $c \neq \lambda^{*}$. 
ЛЕМма 2. Имеет место равенство $u(t, x)=e^{c t} \varphi(x) w(t, x)$, әде $w(t, x)$ - решение уравнения

$$
\partial_{t} w=L w \quad c \text { оператором } \quad L=\frac{1}{2} \Delta+(\nabla \log \varphi) \nabla .
$$

ДокАЗАТЕльСтво. Достаточно подставить функцию $u(t, x)=e^{c t} \varphi(x) w(t, x)$ в уравнение (2) и воспользоваться тем фактом, что $\varphi$ - собственная функция оператора $\frac{1}{2} \Delta+v$ с собственным значением $c$.

ЛЕмма 3. Пусть $w$-решение уравнения (4) с некоторым непрерывным начальным условием $f$. Тогда

1) $w(t, x) \rightarrow c$ при $t \rightarrow \infty$ равномерно по $x$; если $f>0$, то $c \neq 0$;

2) $\nabla w(t, x) \rightarrow 0$ при $t \rightarrow \infty$ равномерно по $x$;

3) если $f>0$, то $\nabla \log w(t, x) \rightarrow 0$ при $t \rightarrow \infty$ равномерно по $x$.

ДоКАЗАТЕЛЬСтво. 1) Пусть $\left(y_{t}\right)$ - случайный процесс на $M$ с генератором $L$ с началом в точке $x \in M$. Обозначим символом $P_{x}$ его закон распределения. Так как $\left(y_{t}\right)$ - диффузионный процесс на окружности, он обладает инвариантной мерой $\mu$ такой, что $\mu(M)=1$ (см., например, [3]). Тогда по теореме 3.4 из [5] мы имеем

$$
w(t, x) \rightarrow \int_{M} f(x) \mu(d x)=c,
$$

и $c>0$ для положительных $f$, поскольку мера $\mu$ положительна.

Чтобы доказать, что эта сходимость является равномерной, зафиксируем какое-нибудь $x_{0} \in M$. Пусть $\tau$ - марковский момент времени, когда случайный процесс $\left(y_{t}\right)$ приходит в точку $x_{0}$. Обозначим $g(t, x)=P_{x}(\tau>t)$ и заметим, что функция $g$ непрерьвна и $g\left(t_{2}, x\right) \leqslant g\left(t_{1}, x\right)$ при $t_{2}>t_{1}$. По лемме 5.2 из [5] случайный процесс $\left(y_{t}\right)$ является возвратньм, так как уравнение $L u=0$ имеет единственное ограниченное решение на произвольном интервале $[a, b] \subset \mathbb{R}$ с заданньми граничными условиями. Следовательно, $g(t, x) \rightarrow 0$ при $t \rightarrow \infty$, и из монотонности функции $g$ следует, что эта сходимость равномерна по $x$.

Далее, мы имеем $w(t, x)=\mathbb{E}_{x} f\left(y_{t}\right)$. Пусть $\varepsilon>0$; вследствие уже доказанной поточечной сходимости найдется число $T_{1}>0$ такое, что для любого $t>T_{1}$ выполняется

$$
\left|w\left(t, x_{0}\right)-c\right|<\frac{\varepsilon}{2}
$$

Вследствие равномерной сходимости $g(t, x)-P_{x}\{\tau>t\} \rightarrow 0$ найдется число $T_{2}>0$ такое, что для любого $t>T_{2}$ и для любого $x$ выполняется

$$
P_{x}\{\tau>\log t\}<\frac{\varepsilon}{2(c+\|f\|)} .
$$

Наконец, найдется число $T_{3}>0$ такое, что для любого $t>T_{3}$ выполняется $t-\log t>T_{1}$. Пусть $T_{0}=\max \left\{T_{1}, T_{2}, T_{3}\right\}$. Обозначим плотность распределения случайной величины $\tau$ символом $p_{x}$. Тогда мы имеем для всех $t>T_{0}$ и для всех $x$

$$
\begin{aligned}
|w(t, x)-c| & =\left|\mathbb{E}_{x} \mathbf{1}_{\{\tau>\log t\}} f\left(y_{t}\right)+\int_{0}^{\log t} p_{x}(\theta) \mathbb{E}_{x_{0}} f\left(x_{t-\theta}\right) d \theta-c\right| \\
& \leqslant(\|f\|+c) P_{x}\{\tau>\log t\}+\int_{0}^{\log t} p_{x}(\theta)\left|w\left(t-\theta, x_{0}\right)-c\right| d \theta<\varepsilon
\end{aligned}
$$

что влечет равномерность сходимости $w(t, x) \rightarrow c$ при $t \rightarrow \infty$.

2) Пользуясь предыдушим утверждением, мы имеем

$$
\partial_{t} w(t, x)=\left(L e^{t L} f\right)(x)=\left(e^{t L} L f\right)(x) \rightarrow c^{\prime}
$$


равномерно по $x$. Так как кроме этого выполняется также $w(t, x) \rightarrow c$, мы получаем $c^{\prime}=0$. После умножения на $2 \varphi^{2}$ уравнение (4) переписывается в виде $\nabla\left(\varphi^{2} \nabla w\right)=2 \varphi^{2} \partial_{t} w$. Следовательно,

$$
\nabla w(t, x)=c(t) \varphi^{-2}(x)+2 \varphi^{-2}(x) \int_{x_{0}}^{x} \varphi^{2}(y) \partial_{t} w(t, y) d y
$$

где $c(t)$ - некоторая функция. Используя дополнительное условие периодичности

$$
\int_{M} \nabla w(t, x) d x=0
$$

для каждого $t$, ограниченность функции $\varphi$ и ее отделенность от нуля, а также равномерную сходимость $\partial_{t} w(t, y) \rightarrow 0$, мы получаем $c(t) \rightarrow 0$ и $\nabla w(t, x) \rightarrow 0$ при $t \rightarrow \infty$ равномерно по $x$.

3) Это утверждение следует из двух предыдущих.

Теорема 2. Семейство поверхностных мер $\mathbb{S}_{T}$ при $T \rightarrow \infty$ слабо сходится $\kappa$ закону распределения броуновского движения со сносом $\left(y_{t}\right)$, которое является решением стохастического дифференциального уравнения

$$
d y_{t}=d b_{t}+\nabla \log \varphi\left(y_{t}\right) d t
$$

с начальным условием $y_{0}=a_{0}$.

ДокАЗАТЕЛЬСтво. По теореме 1 для каждого $T>0$ случайный процесс $\left(y_{t}^{T}\right)$, соответствующий мере $\mathbb{S}_{T}$, является решением уравнения (1). Покажем, что коэффициенты уравнения (1) сходятся к коэффициентам уравнения (5) в том смысле, в каком это требуется в условиях теоремы 11.1.4 из [6]. Согласно леммам 2 и 3 мы получаем

$$
\nabla \log u(t, x)=\frac{\nabla\left[e^{c t} \varphi(x) w(t, x)\right]}{e^{c t} \varphi(x) w(t, x)}=\nabla \log \varphi(x)+\nabla \log w(t, x) \rightarrow \nabla \log \varphi(x)
$$

при $t \rightarrow \infty$ равномерно по $x$. Следовательно, функция $\nabla \log u(t, x)$ равномерно ограничена для всех $t$ и $x$. Далее, для каждого $T_{0}>0$ выполняется

$$
\int_{0}^{T_{0}} \sup _{x \in M}|\nabla \log u(T-t, x)-\nabla \log \varphi(x)| d t=\int_{T-T_{0}}^{T} \sup _{x \in M}|\nabla \log w(t, x)| d t \rightarrow 0 .
$$

Таким образом, оба условия теоремы 11.1 .4 из [6] выполнены и, следовательно, $\mathbb{S}_{T} \rightarrow \mathscr{L}(y)$ в смысле слабой сходимости.

Автор выражает глубокую благодарность профессору О.Г. Смолянову за внимание к работе.

\section{СПИСОК ЦИТИРОВАННОЙ ЛИТЕРАТУРЫ}

1. Смолянов О.Г. // Докл. РАН. 1995. Т. 345. №4. С. 455-458. 2. Sidorova N. А., Smolyanov O. G., von Weizsäcker H., Wittich O. // J. Funct. Anal. 2004. V. 206. № 2. P. 391-413. 3. Pinsky R. G. Second order elliptic operators with periodic coefficients: criticality theory, perturbations, and positive harmonic functions // J. Funct. Anal. 1995. V. 129. P. 80-107. 4. Agmon S. On positive solutions of elliptic equations with periodic coefficients in $\mathbb{R}^{n}$, spectral results and extensions to elliptic operators on Riemannian manifolds / / Differential Equations. Birmingham, 1983. 5. Хасьминский Р. З. Эргодические свойства возвратных диффузионных процессов и стабилизация решений параболических уравнений // Теор. вероятн. и ее примен. 1960. Т. 5. № 2. C. 179-196. 6. Stroock D. W., Varadhan R. S. R. Multidimensional Diffusion Processes. Grundlehren der mathematischen Wissenschaften. Berlin-Heidelberg-New York: Springer-Verlag, 1979. 